\title{
Adquisición de competencias a través de la simulación y juego de rol en el área contable
}

\author{
María Isabel Ros Clemente \\ Universidad Católica San Antonio de Murcia \\ irclemente@ucam.edu \\ María del Carmen Conesa PÉREZ \\ Universidad Católica San Antonio de Murcia \\ ccperez@ucam.edu
}

Recibido: 06/11/2012

Aceptado: 23/01/2013

\begin{abstract}
Resumen
El EEES pretende que el alumno adquiera las competencias propias del perfil profesional de la titulación que estudien, luego la tarea a realizar por el profesor es adaptar el proceso de enseñanza, y por tanto su metodología, a la adquisición de las mismas e incorporar modelos didácticos centrados en la práctica que potencien una formación en competencias. Se pretende analizar la eficiencia del aprendizaje experimental y experiencial en el área contable, mediante la aplicación de un juego de simulación empresarial a dos grupos de alumnos del Grado en ADE. Los resultados obtenidos confirman la efectividad de dichas técnicas en la adquisición de competencias.

Palabras clave: aprendizaje experiencial y experimental, competencias, metodología cooperativa, innovación docente.

\section{Acquisition of Skills through Simulation and Role Play in the Area of Accounting}

\begin{abstract}
The EHEA aims at students to acquire the fundamental skills for a professional qualification to study, hence the task to be performed by instructors would be to adapt the education process, and therefore its methodology, towards acquisition as well as to incorporate practice-centered didactic models for training to enhance skills. We aim to analyze the efficiency of experimental and experiential learning in the area of accounting, by applying a business simulation game to two groups of students of the Degree in Business Administration (ADE). Results obtained already confirm the effectiveness of these techniques in the acquisition of skills.

Keywords: experimental and experiential learning, skills, cooperative methodology, teaching innovation

\section{Referencia normalizada}

ROS CLEMENTE, María Isabel y CONESA PÉREZ, María del Carmen (2013): “Adquisición de competencias a través de la simulación y juego de rol en el área contable". Estudios sobre el Mensaje Periodístico. Vol. 19, Núm. especial marzo, págs.: 419-428. Madrid, Servicio de Publicaciones de la Universidad Complutense.
\end{abstract}

Sumario: 1. Introducción. 2. Metodología. 3. Resultados. 4. Conclusión. 5. Referencias bibliográficas.

\section{Introducción}

En una sociedad globalizada, la capacidad de análisis crítico y de aplicación del conocimiento ocupa un lugar predominante sobre la mera adquisición de conceptos teóricos. Se hace necesario una revisión de las metodologías docentes empleadas para responder a los cambios propuestos en el Espacio Europeo de Educación Superior (EEES). 
Ampliar y readaptar las metodologías docentes a las nuevas demandas del entorno profesional (Aymerich y Gras, 2009) es el nuevo reto de la educación superior. Los métodos y procedimientos didácticos no deben estar enfocadas a la transmisión de información sino a la promoción de competencias en los individuos (Romero, 2010). Se deben adecuar los métodos de enseñanza y aprendizaje al objetivo de adquisición de las competencias vinculadas al perfil o perfiles profesionales de una titulación (De Miguel, 2006b). La tarea del profesor pasa de ser transmisor de conocimientos a gestor del proceso de aprendizaje de los estudiantes, deberá elegir entre las distintas técnicas de aprendizaje dependiendo del tipo de competencias a desarrollar, las características del grupo y las posibilidades que nos ofrece el escenario donde vamos a realizar la actividad (De Miguel, 2006a).

Para titulaciones universitarias relacionadas con la empresa se ha de desarrollar en los alumnos, competencias específicas, genéricas y transferibles que lo capaciten como una persona práctica, con el carácter de un experto, que tenga habilidades claras y experimentadas en su campo, y que gestione con criterios profesionales. Todo ello, solo se podrá adquirir con la aplicación de técnicas o herramientas que apliquen los conocimientos a la práctica.

Se trata de incorporar a la práctica docente de educación superior, un aprendizaje experiencial (basado en experiencias) y un aprendizaje experimental (basado en prácticas experimentales) como estrategias metodológicas para afrontar las nuevas demandas formativas. Dentro del aprendizaje a través de experiencias podemos incluir como técnica, el juego de rol (Ortiz et al., 2010,) e incluir la simulación como técnica experimental (Martín et al., 2007).

El aprendizaje experiencial (Romero, 2010), como estrategia metodológica permite afrontar nuevas demandas formativas, enfocadas al desarrollo de competencias. A su vez promueve la capacidad de aprender a aprender (Department for Education and Skills, 2006) e involucra al individuo en una interacción directa con aquello que estudia (Smith, 2001). El aprendizaje experimental permite, a su vez, mejorar los comportamientos para trabajar en equipo (Martín et al., 2007) y con ello mejorar sus conocimientos, habilidades y destrezas ${ }^{1}$.

Estas herramientas se posicionan en una postura pedagógica constructivista, la orientación de estas técnicas está dirigida más al proceso que al producto, y a través de una experiencia objetiva o subjetiva se intenta desarrollar en los alumnos un razonamiento inductivo (Torres, 1999). El juego de rol y las simulaciones potencian habilidades tales como: el trabajo en equipo, la resolución de conflictos y la toma de decisiones, esenciales todas ellas para desenvolverse en el ámbito empresarial actual (Ortiz et al., 2010). El "juego de rol" por otro lado permite un enfoque de aprendizaje colaborativo. Es una herramienta con la que todos los miembros participan activamente con una simulación de un grupo. Esta simulación permite aproximarse a la realidad (Ortiz et al., 2010). Por otro lado, las "simulaciones" son experiencias que pretenden que el alumno, a través de experiencias concretas, adquieran conceptos, habilidades y competencias, propias del

1 Ver trabajos de Chen, Donahue y Klimoski (2004) o Ellis, Bell Ployhart, Hollengbeck e Ilgen (2005). 
perfil profesional definido en su titulación, todo ello minimizando los riesgos de la práctica en situaciones reales (Ingram y Jackson, 2004).

En el área contable, la aplicación de la técnica de simulación de una empresa, donde los alumnos adquieren el rol de contables o empresarios, permite la adquisición de competencias propias del perfil de la titulación de ahí que el objetivo de este trabajo sea la valoración de dicha técnica.

Específicamente este trabajo persigue analizar, partiendo de dos escenarios diferentes, varios objetivos: si el grado de satisfacción de los alumnos por el uso de esta metodología es elevado, si el alumno percibe una adecuada adquisición de las competencias y la valoración de una serie de características asignadas al juego. Se comprobará la existencia o ausencia de relación entre el índice y esas características. Y por último, analizaremos si las diferencias surgidas entre los grupos son significativas.

\section{Metodología}

El uso del juego de rol y simulación ha sido desarrollado en dos asignaturas del área contable, Contabilidad Financiera y Análisis de los Estados Contables, ambas del grado en Administración y Dirección de empresas.

En esta área de conocimiento se hace imprescindible saber elaborar e interpretar una información económica financiera fundamental para la correcta toma de decisiones, las Cuentas Anuales.

La asignatura de Contabilidad Financiera tiene como objetivo proveer a los alumnos de los conocimientos básicos de contabilidad que le permitan el registro de las principales problemáticas contables y confeccionar las Cuentas Anuales de una empresa según lo establecido en el Plan General de Contabilidad.

El Análisis de Estados Contables permite que los alumnos, partiendo de los conocimientos contables adquiridos previamente, conozcan las herramientas del análisis económico financiero y adquieran la capacidad de analizar e interpretar sus resultados.

En nuestro experimento se aplican dos juegos distintos a dos grupos de alumnos, donde cada uno de ellos manifiesta características y ámbitos diferentes. La simulación propuesta consiste en la aplicación del análisis económico financiero, a través de la creación de una empresa en la que se analizan sus estados contables.

Los alumnos del primer grupo, Simulación Empresarial (SE), son de primero de grado en ADE. La práctica propuesta persigue facilitar al alumno el aprendizaje de la elaboración de esos estados contables. Consiste en un juego de rol y simulación empresarial sobre el registro contable que una empresa lleva a cabo en el desarrollo de su actividad económica durante un ejercicio. Elaborando a 31 de diciembre dos de los cinco estados que configuran las Cuentas Anuales, Balance de Situación y Cuenta de Pérdidas y Ganancias.

El segundo grupo, Juego de Emprendedores (JE) recoge alumnos de segundo de grado en ADE. La simulación propuesta consiste en la aplicación del análisis económico financiero, a través de la creación de una empresa en la que se analizan sus estados contables.

Con la intención de evaluar la eficacia del juego durante el curso 2011-2012, al terminar las asignaturas se pasó una encuesta a una muestra de 51 alumnos ( 25 del juego de simulación empresarial y 26 del juego de emprendedores). 
La encuesta ${ }^{2}$ utilizada se divide en cuatro partes. La primera de ellas hace referencia a información personal del alumno, sexo y edad. La segunda parte pretende que el alumno valore, entre 1 (muy en desacuerdo) y 5 (muy de acuerdo), diversos aspectos del aprendizaje mediante el juego de rol y simulación empresarial. En la tercera parte se pregunta sobre la eficacia del juego pidiéndoles que valoren hasta qué punto el mismo favorece el desarrollo de una serie de competencias. Y en la cuarta y última parte, se puntúan una serie de características del juego.

En primer lugar con los datos obtenidos en la segunda parte de la encuesta se ha desarrollado un índice de satisfacción media, a partir de la valoración dada a 29 items para cada uno de los miembros de la muestra.

Se han calculado los estadísticos descriptivos para cada una de las variables analizadas (índice de satisfacción, competencias generales, competencias específicas y características del juego) estudiando entre otros: el valor máximo y mínimo alcanzado por las variables, el valor que como media más se adopta y su desviación típica, con la finalidad de valorar la metodología aplicada.

Profundizando en el análisis estadístico de los datos, se ha llevado a cabo un estudio exploratorio de las relaciones entre el índice de satisfacción y las variables que recogían distintas características del juego.

Las relaciones entre el índice y las variables cuantitativas que representan las características se analizarán utilizando el coeficiente de correlación lineal de Pearson.

No intentamos explicar la variable dependiente, índice de satisfacción, por medio de regresores, sino sólo describiendo el signo (+/-) de sus relaciones. Por esta razón, se ha aplicado el análisis de correlaciones en vez de la regresión.

Por último, también se han analizado las relaciones entre las competencias específicas adquiridas y las características del juego.

\section{Resultados}

De la información extraída de la primera parte de la encuesta encontramos que la muestra está compuesta por el doble de hombres (66.7\%) que de mujeres (33.3\%).

En cuanto a su edad el $32.5 \%$ se sitúa en la franja de de 18 a 20 años y el $27.5 \%$ supera los 24 años.

Una vez elaborado el índice de satisfacción e interpretando los datos obtenidos observamos que los alumnos alcanzan como media un grado de satisfacción de 4,027 puntos. La variabilidad se recoge por la desviación estándar de la variable Índice de satisfacción (W1), respondiendo a un 12,18\% de la media. En general la situación es favorable hacia la técnica de aprendizaje utilizada.

Tomando en consideración ítems concretos, los más valorados de media, con un 4,196 puntos, han sido los aspectos P.7, es decir, el juego de simulación/rol puede ser útil como complemento de otros métodos de enseñanza alternativos (estudios de casos, lección magistral y libros de texto) y P.34, en el juego de simulación/rol, el esfuerzo personal es necesario para conseguir un adecuado aprendizaje.

${ }^{2}$ Encuesta elaborada a partir de la desarrollada por Carmona, 2008. 
El aspecto del aprendizaje menos valorado de media $(3,824)$, pero con una elevada variabilidad respecto a la misma $28.51 \%$, ha sido en el juego, la cohesión del grupo es importante para obtener unos buenos resultados. El alumno valora los conocimientos del grupo más que la relación personal entre los miembros del mismo, como se aprecia en la valoración de P.28 $(4,020)$ los conocimientos del grupo son importantes para obtener buenos resultados.

Analizando la distribución de frecuencias del índice vemos que el 98\% de los alumnos encuestados puntúan la experiencia por encima del tres.

Al desagregar el índice por muestras según las distintas experiencias de simulación observamos que en la práctica de simulación empresarial (SE) se ha concentrado un mayor número de alumnos en el intervalo 3-4 (56\%). En cambio el 84.6\% del alumnado de la muestra dos ha puntuado el juego de emprendedores entre un 4 y un 5.

Estudiando los resultados obtenidos en la valoración de adquisición de competencias comprobamos que en promedio se puntúa con 3,954 puntos, siendo la variabilidad de $12.98 \%$. Así un $42.9 \%$ puntúa por encima del 4 la adquisición de competencias generales tras la finalización del juego. De nuevo, volvemos a comprobar, que para el grupo JE el porcentaje de alumnos que puntúan por encima del 4 es superior a los del grupo SE.

Del estudio de la adquisición de competencias específicas advertimos que en SE entre el $70-80 \%$ de los alumnos opinan que está técnica de aprendizaje les ha permitido la adquisición de las competencias específicas asignadas. El resultado obtenido en el grupo JE es aún más satisfactorio, entre el 90 y $100 \%$ de los alumnos están muy satisfechos con la adquisición de las competencias asignadas.

Analizando las puntuaciones obtenidas de media por cada una de las seis características estudiadas observamos los siguientes resultados (ver tabla 1):

Tabla 1. Características. Ambos grupos (\%)

\begin{tabular}{|l|c|c|c|c|c|c|}
\hline Valor & Y1 & Y2 & Y3 & Y4 & Y5 & Y6 \\
\hline 1 & 2,0 & 2,0 & & 2,1 & & \\
\hline 2 & 2,0 & 8,2 & & 6,3 & 4,1 & 2,0 \\
\hline 3 & 10,2 & 30,6 & 28,6 & 16,7 & 14,3 & 16,3 \\
\hline 4 & 32,7 & 38,8 & 49,0 & 43,8 & 61,2 & 44,9 \\
\hline 5 & 53,1 & 20,4 & 22,4 & 31,3 & 20,4 & 36,7 \\
\hline TOTAL & 100 & 100 & 100 & 100 & 100 & 100 \\
\hline
\end{tabular}

Fuente: Elaboración propia

En la característica Participación activa del alumno (Y1) el 85.8\% considera que ha participado activamente en este proceso de aprendizaje. Una vez más al desagregar los resultados por muestras observamos que la distribución de frecuencias en el grupo JE es más asimétrica hacia la izquierda, concentrando el $96.1 \%$ en valores por encima del 4 y un $0 \%$ por debajo de 3 .

En Diversión (Y2) el 59.2\% se ha divertido mucho con el desarrollo de esta actividad experiencial y experimental. Si bien la puntación media obtenida por esta característica es la más baja de todas (3.674) y su variabilidad respecto a esa puntuación 
es la más alta con un $26.29 \%$. Esta característica presenta un elevado porcentaje de indiferencia, e igual para ambos grupos. Mientras que el grupo JE la distribución entre las puntuaciones 4 y 5 es similar, en el grupo SE la frecuencia es superior para la valoración 4 con una diferencia de 34.8 puntos porcentuales respecto a la valoración 5 .

Nuestro estudio corrobora lo dicho por otros autores (Aymerich y Gras, 2009; De Miguel, 2006b) en cuanto a la Complementariedad con otros métodos docentes (Y3). Ninguno de los alumnos piensa que esta metodología de aprendizaje no aporte nada, no hay puntuaciones del $1 \mathrm{al} 3$, sino que ven que no es incompatible con otros métodos. El $71.4 \%$ considera estas técnicas como un buen complemento docente. En la distinción por grupos observamos la misma dinámica que en otras variables. Para el grupo JE el 88.4\% de alumnos se concentran en los valores 4 y 5 , siendo del $52.2 \%$ en el caso del grupo SE, que a su vez presenta el porcentaje más elevado $47.8 \%$ de indiferencia.

El tiempo empleado (Y4) presenta una razonablemente buena puntuación media 3.958 , sin embargo, su variabilidad respecto a la media se cifra en un $24,042 \%$. Al estudiar la distribución de frecuencias observamos que el $8.4 \%$ en la muestra total opina que el tiempo no fue el adecuado. Frente al $75.1 \%$ que piensan que es apropiado. Por grupos, es en SE donde se produce una mayor distribución en los distintos valores.

Con el estudio de la siguiente característica pretendemos comprobar si la complejidad (Y5) de los juegos aplicados se adapta a los conocimientos de nuestros alumnos. Esta variable obtiene una alta puntuación como media 4,225 puntos, con una desviación típica de 0,803 . Aunque la desviación es más alta que en otras características, la valoración es buena, lo que significa que los alumnos consideran que el juego presenta una adecuada complejidad dados sus conocimientos.

En Cercanía a la realidad (Y6) nuestro experimento obtiene una puntuación satisfactoria de 4,163 $( \pm 0,77)$ sobre 5, por lo que las simplificaciones hechas en el juego no se consideran excesivas ni alejadas de la realidad.

Examinando la relación de las características con el índice de satisfacción global, la tabla 2 recoge la correlación del índice con todas las demás variables

Tabla 2. Correlaciones del índice con las características

\begin{tabular}{|c|c|c|c|c|c|c|}
\hline & 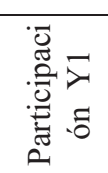 & $\begin{array}{l}: \frac{0}{0} \\
\frac{2}{0} \\
0 \\
0 \\
0\end{array}$ & 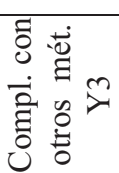 & $\stackrel{̊}{\stackrel{\Xi}{\Xi}}$ &  & 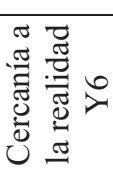 \\
\hline \multirow{2}{*}{ Índice Satisfacción } & 0.6343 & 0.3776 & 0.3915 & 0.6209 & 0.3534 & 0.5428 \\
\hline & 0.0000 & 0.0081 & 0.0059 & 0.0000 & 0.0137 & 0.0001 \\
\hline
\end{tabular}

Fuente: Elaboración propia

Observamos que el índice tiene una muy alta correlación (significativa 0.000) con participación activa del alumno, tiempo empleado y cercanía a la realidad. Estableciéndose una relación positiva entre estas características y el índice. Por lo que, en principio, deberíamos esperar que cuanto más aumente la participación del alumno en el juego, más se adecue el tiempo empleado al mismo y los supuestos del juego sean lo más realistas posibles mayor será la satisfacción del alumno con el aprendizaje obtenido mediante la realización del experimento/experiencia. 
También, significativamente distinta de cero encontramos la correlaciones entre el índice y la característica de diversión e igualmente la relación es positiva, al aumentar la diversión aumenta el aprendizaje del alumno.

La correlación obtenida entre el índice de satisfacción y la complejidad nos muestra que si la práctica se adecua en complejidad al nivel de conocimientos de los alumnos se aumenta el grado de satisfacción.

Observamos que aparece una relación negativa entre el número de componentes del grupo y el índice, interpretándose que a mayor número de componentes en el grupo menor es la satisfacción con la técnica de aprendizaje. Con la variable sexo, la correlación no es significativamente distinta de cero por lo que no existe relación entre el índice de satisfacción y la variable sexo. En cambio, si aparece relación directa positiva con la variable edad.

Por último, si correlacionamos las características con la adquisición de competencias específicas nos percatamos de que la adquisición de competencias en el grupo SE está principalmente relacionada con la participación activa del alumno y con cercanía a la realidad, siendo la correlación de las distintas competencias específicas con esas dos características significativamente distinta de cero. Interpretándose que a mayor participación de los alumnos y más realistas los supuestos mayor es el número de competencias adquiridas.

De las correlaciones entre competencias y características para JE destacamos especialmente las de las características de diversión y adecuación del tiempo que son las que aparecen correlacionadas positivamente con la mayoría de las competencias.

En la evaluación de las prácticas, hemos apreciado, que si bien los resultados son muy positivos en ambos grupos, los del juego de emprendedores son superiores. Consideramos interesante indagar si las diferencias son significativas mediante un test de igualdad de medias.

Tabla 3. Test de igualdad de medias para los índices de satisfacción y características

\begin{tabular}{|l|l|l|l|l|}
\hline & $\mathrm{t}(\mathrm{a})$ & $\mathrm{p}$-value & $\mathrm{t}(\mathrm{b})$ & $\mathrm{p}$-value \\
\hline Índice de satisfacción general & -4.03 & 0.00 & -3.98 & 0.00 \\
\hline Competencias & & & & \\
\hline Generales & -3.59 & 0.00 & -3.53 & 0.00 \\
\hline Específicas & -4.43 & 0.00 & -4.34 & 0.00 \\
\hline Características & & & & \\
\hline Participación activa & -2.91 & 0.01 & -2.81 & 0.01 \\
\hline Diversión & -1.98 & 0.05 & -1.97 & 0.06 \\
\hline Complementariedad & -3.32 & 0.00 & -3.32 & 0.00 \\
\hline Tiempo empleado & -2.19 & 0.03 & -2.13 & 0.04 \\
\hline Motivación & -2.4 & 0.02 & -2.35 & 0.02 \\
\hline Complejidad & -1.42 & 0.16 & -1.39 & 0.17 \\
\hline Cercanía a la realidad & -2.65 & 0.01 & -2.59 & 0.01 \\
\hline
\end{tabular}

a) Bajo el supuesto de varianzas iguales

b) Bajo el supuesto de varianzas diferentes

c) La hipótesis nula es que las medias poblacionales son iguales

Fuente: Elaboración propia 
Según los resultados ofrecidos por el estadístico t bajo la hipótesis nula de igualdad de medias, la tabla 3muestra que existen diferencias significativas en la valoración que dan ambos grupos a la satisfacción general y a la adquisición de competencias generales y específicas. Además, en cuanto a las características las diferencias son significativas en todas ellas excepto en diversión y complejidad.

Creemos que esas diferencias pueden deberse a la mayor edad, madurez y grado de conocimientos sobre la materia que tienen los alumnos del grupo dos, juego de emprendedores. No obstante, sería necesario llevar a cabo un estudio más pormenorizado para indagar en las causas de esas diferencias.

\section{Conclusiones}

La aplicación de técnicas de aprendizaje experimental y experiencial parecen ser las adecuadas para conseguir un aprendizaje efectivo y eficaz, dado la alta valoración de estas metodologías, un 4,027 puntos sobre 5 .

Hemos mostrado de forma práctica que estos juegos son útiles para el desarrollo de competencias por parte de los alumnos ya que más del $70 \%$, del $90 \%$ en JE, considera que la adquisición de competencias generales y específicas es satisfactoria o muy satisfactoria con la utilización de ésta metodología.

Tras el análisis realizado a las seis características seleccionadas (participación activa del alumno, diversión, complementariedad, tiempo empleado, complejidad y cercanía a la realidad) se pone de manifiesto la alta puntuación de cada una de ellas obtenidas en nuestro juego.

Estudiada la relación entre la satisfacción con los distintos aspectos del aprendizaje y cada una de las características, se observa que: todas las características están significativamente correlacionadas con la efectividad del juego, por tanto, cuanto más participativo, divertido, cercano a la realidad, y adecuado en tiempo y complejidad sea el juego, mayor será la satisfacción del alumno hacia la efectividad del mismo en su aprendizaje.

La adquisición de competencias específicas es mayor cuanto más participativa y cercana a la realidad es la metodología adoptada.

En la evaluación de las prácticas, hemos apreciado, que si bien los resultados son muy positivos en ambos grupos, los del juego de emprendedores son superiores. Realizado el test de igualdad de medias y obteniendo diferencias significativas entre ambos grupos, consideramos interesante llevar a cabo una investigación adicional para indagar las causas que provocan esas diferencias.

Desde nuestro punto de vista se necesitaría una investigación adicional para ampliar nuestra muestra, permitiendo así generalizar nuestras afirmaciones.

\section{Referencias bibliográficas}

AYMERICH, María y GRAS, Ma Eugenia (2009): "Las metodologías docentes y su valoración por parte de los estudiantes universitarios". Ponencia presentada en el II Congreso Internacional UNIVEST, Gerona.

CARMONA MARTINEZ, Mercedes (2010):'Encuesta de valoración de la efectividad en el aprendizaje de un juego de simulación empresarial". Comunicación personal. 
CHEN, Gilad, DONAHUE, Lisa y KLIMOSKI, Richard (2004): “Training undergraduates to work in organizational teams". Academy of Management Learning and Education, 3 (1), 27-40.

DE MIGUEL, Mario (2006a): "Metodologías para optimizar el aprendizaje. Segundo objetivo del Espacio Europeo de Educación Superior". Revista Interuniversitaria de Formación del Profesorado, 20 (3), 71-91.

DE MIGUEL, Mario (2006b): Metodologías de enseñanza y aprendizaje para el desarrollo de competencias. Madrid, Alianza Editorial.

DEPARTAMENT FOR EDUCATION AND SKILLS (2006): "Learning Outside the Classroom Manifesto". Nottingham, DfES Publications.

ELLIS, Aleksander, BELL, Bradford, PLOYHART, Robert, HOLLENBECK, John e ILGEN, Daniel (2005): “The impact of task-and team-generic teamwork skills training on team effectiveness". Working Paper, Cornell University, School of Industrial and Labor Relations, Center for Advanced Human Resource Studies.

INGRAM, Kathkeen y JACKSON, Katherine (2004): "Simulations as authentic learning strategies: Bridging the gap between theory and practice in performance technology". Association for educational communications and technology (No. de servicio de reproducción de documentos ERIC ED485146) en Hernández, M. et al. (2011).

MARTÍN PERÉZ, Victor; MARTÍN CRUZ, Natalia; y PERÉZ SANTANA, Ma Pilar (2007): El uso de las nuevas tecnologías para favorecer el trabajo en equipo. La simulación estratégica como técnica de aprendizaje experimental. Disponible: $\mathrm{http} / / /$ dialnet.unirioja.es/servlet/oaiart? codigo $=2234390$. [Consultado el día $22 \mathrm{de}$ julio de 2012]

ORTIZ DE URBINA CRIADO; Marta, MEDINA SALGADO, Sonia; y DE LA CALLE DURAN, Carmen (2010): "Herramientas para el aprendizaje colaborativo: Una aplicación práctica del juego de rol". TESIS, 11 (3), 277-301.

ROMERO ARIZA, Marta (2010): "El aprendizaje experiencial y las nuevas demandas formativas". Revista de Antropología Experimental, 10, 89-102.

SMITH, Mark K. (2001): "David a Kolb on experiential learning". The Encyclopedia of Informal Education. Disponible en: http://www.seer.org/pages/csap.pdf [Consultado el 28 de julio de 2012]

TORRES, Jorge (1999): "Programa "caso simulado" una tecnología educativa para el apoyo didáctico". Disponible: http://148.213.1.36/Documentos/Encuentro/PDF /68.pdf [Consultado el 25 de julio de 2012] 


\section{María Isabel ROS CLEMENTE}

Universidad Católica San Antonio de Murcia (UCAM)

Facultad de Ciencias Jurídicas y de la Empresa

irclemente@ucam.edu

Profesora en Administración y Dirección de Empresas de la UCAM

Miembro del Grupo de Investigación "Organización de empresas y marketing”

\section{María del Carmen CONESA PÉREZ}

Universidad Católica San Antonio de Murcia (UCAM)

Facultad de Ciencias Jurídicas y de la Empresa

ccperez@ucam.edu

Profesora en Administración y Dirección de Empresas de la UCAM

Miembro del Grupo de Investigación "Organización de empresas y marketing” 